\title{
On a class of spatial discretizations of equations of the nonlinear Schrödinger type
}

\author{
P.G. Kevrekidis ${ }^{a}{ }^{a}$, S.V. Dmitriev ${ }^{b}$, A.A. Sukhorukov ${ }^{b}$ \\ a Department of Mathematics and Statistics, University of Massachusetts Lederle Graduate Research Tower, Amherst, MA 01003-4515, USA \\ ${ }^{\mathrm{b}}$ Institute of Industrial Science, University of Tokyo, 4-6-1 Komaba, Meguro-ku, Tokyo 153-8505, Japan \\ ${ }^{\mathrm{c}}$ Nonlinear Physics Centre, Research School of Physical Sciences and Engineering, Australian National University, \\ Canberra, ACT 0200, Australia
}

Available online 29 November 2006

\begin{abstract}
We demonstrate the systematic derivation of a class of discretizations of nonlinear Schrödinger (NLS) equations for general polynomial nonlinearity whose stationary solutions can be found from a reduced two-point algebraic condition. We then focus on the cubic problem and illustrate how our class of models compares with the well-known discretizations such as the standard discrete NLS equation, or the integrable variant thereof. We also discuss the conservation laws of the derived generalizations of the cubic case, such as the lattice momentum or mass and the connection with their corresponding continuum siblings.
\end{abstract}

(C) 2006 IMACS. Published by Elsevier B.V. All rights reserved.

PACS: 03.40.Kf; 63.20Pw

\section{Introduction}

In the past few years, the role of spatial discreteness in lattice systems described by differential-difference equations has been increasingly recognized [3]. In these settings, the spatial variables are discrete, while the evolution variable is continuum. Relevant applications are continuously arising in rather diverse physical contexts such as the spatial dynamics of optical beams in coupled waveguide arrays in nonlinear optics [6], the temporal evolution of BoseEinstein condensates (BECs) in optical lattices in soft-condensed matter physics [15], the DNA double strand in biophysics [22], and so on.

In the examples that stem from physical applications, the form of the discrete model is dictated by the underlying physics, and typically that form is the discrete nonlinear Schrödinger (DNLS) equation [16]. However, there are also exceptions to this rule, where the nature of the nonlinearity [12] or of the dispersion [10] or both [18] imposes variations of this ubiquitous model. Another motivation to study such modified DNLS models is a more mathematical one, namely the aim of identifying models with good mathematical properties (e.g., exact solutions, additional symmetries or, possibly, complete integrability). Such a program was initiated by the derivation of an integrable analog of the DNLS equation, namely the so-called Ablowitz-Ladik model [1]. This was later used for computational studies of the

\footnotetext{
* Corresponding author.

E-mail address: kevrekid@math.umass.edu (P.G. Kevrekidis).
} 
NLS equation [2], as well as implemented as a good starting point for developing perturbation theoretic approaches to the DNLS limit, in order to examine the existence and stability of its solutions [13].

We should note here that a similar, mathematically minded program of discretizations has been evolving for KleinGordon (KG) type lattices. For instance, integrable discretizations of equations such as the sine-Gordon model have been obtained [17,19]; there have also been attempts to systematically discretize preserving symmetries of the underlying continuum model, such as, especially, a discrete analog of translational invariance. It has often been noted that "standard" (e.g., centered-difference schemes for Laplacian type operators) discretizations violate translational invariance, leading stable and unstable steady states (typically centered on-site and inter-site between two lattice nodes). This not only imposes undesirable mathematical properties [5], but also modifies the underlying phenomenology in comparison with the continuum model [23]. In that view, discretizations that preserve an effective translational invariance by allowing the center of a stationary state to be a free parameter (rather than to be fixed on- or half-way between two lattice sites) have been sought. In this way, such discretizations also avoid "energy barriers" (so called Peierls-Nabarro barriers) between on-site and inter-site states. Such discrete models have been constructed in the KG case, based on a discretization of the energy, using the Bogomol'nyi approach [25], as well as a discretization of the equation of motion ensuring the persistence of a discrete momentum conservation law $[14,8]$. These classes of models were subsequently tested for the potential bearing of travelling wave solutions, using the technology based on the calculation of the Stokes constant [4]; this led to the conjecture that such models may possess isolated, exact, travelling lattice solutions. It is, finally, worthwhile to note that while motivated by their mathematical properties, such models may also bear physical relevance as is indicated, e.g., in the very recent work of [26] for the sine-Gordon case.

While this technology has been well developed for the single (i.e., scalar) field case of KG lattices, such considerations do not seem to have been applied to NLS type lattices, to the best of our knowledge. The present manuscript aims to partially fill this gap, by presenting a systematic methodology for deriving discretizations of polynomial nonlinearity partial differential equations (PDEs) of the NLS type. The main novel feature of these discrete models is that, contrary to what is the case for the standard DNLS equation, they preserve a discrete analog of the momentum conservation law. In fact, we show that in the cubic case, they are natural homotopic generalizations of the integrable NLS discretization of [1].

Our presentation will be structured as follows. In Section 2, we present the general setup of the continuum and discrete models of the present work. In Section 3, we illustrate the auxiliary problem that aids us to construct the desired discretizations in Section 4. In Section 5, we study some of the conservation laws of the obtained models, while in Section 6, we discuss their solitonic properties. Finally, in Section 7, we briefly summarize our findings and present our conclusions.

\section{Setup}

We present our methodology for the generalized NLS equation of the form

$$
i \psi_{t}+\frac{1}{2} \psi_{x x}+G^{\prime}\left(|\psi|^{2}\right) \psi=0
$$

where $\psi(x, t)$ is a complex function of two real variables; $G(\xi)$ is a real function of its argument and $G^{\prime}(\xi)=\mathrm{d} G / \mathrm{d} \xi$.

We introduce the lattice $x_{n}=n h$, where $h$ is the lattice spacing and $n=0, \pm 1, \pm 2, \ldots$. We also introduce the following shorthand notations

$$
\psi_{n-1}=\psi_{-}, \quad \text { and } \quad \psi_{n+1}=\psi_{+},
$$

and will focus only on discretizations that involve such nearest neighbor sites.

Our more specific aim will be to construct the discrete analogues of Eq. (1) of the form:

$$
i \dot{\psi}_{n}+r\left(\psi_{-}, \psi_{-}^{\star}, \psi_{n}, \psi_{n}^{\star}, \psi_{+}, \psi_{+}^{\star}\right)=0,
$$

such that the ansatz

$$
\psi_{n}(t)=f_{n} \mathrm{e}^{i \omega t},
$$

reduces Eq. (3) to the three-point discrete problem of the form

$$
-\omega f_{n}+R\left(f_{-}, f_{n}, f_{+}\right)=0,
$$


whose solution can be found from a reduced two-point discrete problem $u\left(f_{-}, f_{n}\right)=0$. Such a selection will entail a mono-parametric freedom for the resulting algebraic problem leading to stationary state solutions. This will, in turn, be responsible for the effective translational invariance in what follows.

\section{Auxiliary problem}

Firstly, we formulate an auxiliary problem. Seeking stationary solutions of Eq. (1) in the form

$$
\psi(x, t)=f(x) \mathrm{e}^{i \omega t},
$$

we reduce it to an ordinary differential equation (ODE) for the real function $f(x)$,

$$
D(x) \equiv f^{\prime \prime}-2 \omega f+2 f G^{\prime}\left(f^{2}\right)=0,
$$

having the first integral

$$
u(x) \equiv\left(f^{\prime}\right)^{2}-2 \omega f^{2}+2 G\left(f^{2}\right)=0,
$$

where the integration constant was set to be zero. This choice will be relevant for constructing discretizations supporting localized solutions with appropriate decay conditions at infinity. The case of nonzero integration constant can produce discretizations supporting solutions of different types including periodic solutions [9].

We then identify discretizations of Eq. (7) of the form

$$
D\left(f_{-}, f_{n}, f_{+}\right)=0,
$$

such that solutions to the three-point discrete Eq. (9) can be found from a reduced two-point problem

$$
u\left(f_{-}, f_{n}\right) \equiv \frac{1}{h^{2}}\left(f_{n}-f_{-}\right)^{2}-2 \omega f_{-} f_{n}+2 G\left(f_{-}^{2}, f_{n}^{2}\right)=0,
$$

which is a discrete version of Eq. (8), assuming that $G\left(f_{-}^{2}, f_{n}^{2}\right)$ reduces to $G\left(f^{2}\right)$ in the continuum limit $(h \rightarrow 0)$.

Taking into account that Eq. (7) is the static Klein-Gordon equation with the potential

$$
V(f)=\omega f^{2}-G\left(f^{2}\right),
$$

a wide class of discretizations solving the auxiliary problem has been offered in the very recent work of ref. [8].

For example, discretizing the left-hand side of the identity $(1 / 2) \mathrm{d} u / \mathrm{d} f=D(x)$, we obtain the discrete version of Eq. (7),

$$
D_{1}\left(f_{-}, f_{n}, f_{+}\right) \equiv \frac{u\left(f_{n}, f_{+}\right)-u\left(f_{-}, f_{n}\right)}{f_{+}-f_{-}}=0 .
$$

Formally, $D_{1}\left(f_{-}, f_{n}, f_{+}\right)=0$ is a three-point problem but, clearly, its solutions can be found from the two-point problem $u\left(f_{-}, f_{n}\right)=0$ and thus, the auxiliary problem is solved. We note, in passing, that this type of argument was first proposed in ref. [14].

\section{Main problem}

Coming back to our main problem of finding special discretizations for Eq. (1), we should remark that among the solutions to the auxiliary problem we should select the ones which can be rewritten in terms of $\psi_{n}$ and $\psi_{n}^{\star}$ in the desired form of Eq. (3). This can be done when $D_{1}$ given by Eq. (12) is written in a non-singular form (i.e., if the denominator cancels with an appropriate factoring of the numerator). This always occurs if $G(\xi)$ is polynomial and if $u\left(f_{-}, f_{n}\right)$ possesses the symmetry

$$
u\left(f_{-}, f_{n}\right)=u\left(f_{n}, f_{-}\right) .
$$

We thus focus on $G(\xi)$ in the form of Taylor expansion,

$$
G\left(|\psi|^{2}\right)=\sum_{k=1}^{\infty} a_{k}\left(|\psi|^{2}\right)^{k},
$$


with real coefficients $a_{k}$. As a simplified special example, we showcase the setting where we retain only the first four terms of the expansion (i.e., the summation above goes up to $k=4$ rather than to $k \rightarrow \infty$ ). In the latter case, after substituting Eq. (6), we construct the following general two-point discrete analog

$$
\begin{aligned}
G\left(f_{-}^{2}, f_{n}^{2}\right)= & \frac{a_{1}}{2}\left(f_{-}^{2}+f_{n}^{2}\right)+a_{2}\left[\frac{\alpha}{2}\left(f_{-}^{4}+f_{n}^{4}\right)+(1-\alpha) f_{-}^{2} f_{n}^{2}\right]+\frac{a_{3}}{2}\left[\beta\left(f_{-}^{6}+f_{n}^{6}\right)+(1-\beta) f_{-}^{2} f_{n}^{2}\left(f_{-}^{2}+f_{n}^{2}\right)\right] \\
& +a_{4}\left[\frac{\gamma}{2}\left(f_{-}^{8}+f_{n}^{8}\right)+\frac{\delta}{2} f_{-}^{2} f_{n}^{2}\left(f_{-}^{4}+f_{n}^{4}\right)+(1-\gamma-\delta) f_{-}^{4} f_{n}^{4}\right]
\end{aligned}
$$

where $\alpha, \beta, \gamma$, and $\delta$ are free parameters. In the continuum limit $(h \rightarrow 0)$ one has $f_{-} \rightarrow f_{n}$ and Eq. (15) obtains the form of Eq. (14). The symmetry condition of Eq. (13) is satisfied for $u\left(f_{-}, f_{n}\right)$ given by (10) since in Eq. (15) we have $G\left(f_{-}^{2}, f_{n}^{2}\right)=G\left(f_{n}^{2}, f_{-}^{2}\right)$.

Eq. (12) with $u\left(f_{-}, f_{n}\right)$ given by Eq. (10) and $G\left(f_{-}^{2}, f_{n}^{2}\right)$ given by Eq. (15) assumes the following form

$$
-\omega f_{n}+\frac{1}{2 h^{2}}\left(f_{-}-2 f_{n}+f_{+}\right) \quad+\left(f_{-}+f_{+}\right) R\left(f_{-}^{2}, f_{n}^{2}, f_{+}^{2}\right)=0,
$$

where

$$
\begin{aligned}
R\left(f_{-}^{2}, f_{n}^{2}, f_{+}^{2}\right)= & \frac{a_{1}}{2}+a_{2}\left[\frac{\alpha}{2}\left(f_{-}^{2}+f_{+}^{2}\right)+(1-\alpha) f_{n}^{2}\right]+\frac{a_{3}}{2} \beta\left(f_{-}^{4}+f_{-}^{2} f_{+}^{2}+f_{+}^{4}\right) \\
& +\frac{a_{3}}{2}(1-\beta) f_{n}^{2}\left(f_{-}^{2}+f_{n}^{2}+f_{+}^{2}\right)+a_{4}\left(f_{-}^{2}+f_{+}^{2}\right)\left[\frac{\gamma}{2}\left(f_{-}^{4}+f_{+}^{4}\right)+(1-\gamma-\delta) f_{n}^{4}\right] \\
& +a_{4} \frac{\delta}{2} f_{n}^{2}\left(f_{-}^{4}+f_{n}^{4}+f_{+}^{4}+f_{-}^{2} f_{+}^{2}\right) .
\end{aligned}
$$

One can conclude that the discretization of NLS equation in the form:

$$
i \dot{\psi}_{n}+\frac{1}{2 h^{2}}\left(\psi_{-}-2 \psi_{n}+\psi_{+}\right)+\left(\psi_{-}+\psi_{+}\right) R\left(\left|\psi_{-}\right|^{2},\left|\psi_{n}\right|^{2},\left|\psi_{+}\right|^{2}\right)=0
$$

with $R$ given by the expression of (17) satisfies the generalized Eq. (1) with $G(\xi)$ given by Eq. (14). However, as per the construction above, additionally, the corresponding discrete equation for the stationary solutions of the form of Eq. (4) is the three-point problem of Eq. (16), whose solution can be found through the two-point reduction of Eq. (10) where $G\left(f_{-}^{2}, f_{n}^{2}\right)$ is given by Eq. (15).

It is interesting to note that the "standard" DNLS equation

$$
i \dot{\psi}_{n}+\frac{1}{2 h^{2}}\left(\psi_{-}-2 \psi_{n}+\psi_{+}\right)+\left|\psi_{n}\right|^{2} \psi_{n}=0,
$$

does not belong to the above class and more generally does not share the reduction property used above. Instead, and focusing only on the cubic Kerr nonlinearity (where $G^{\prime}$ is linear in its argument), we obtain from Eqs. (18) and (17)

$$
i \dot{\psi}_{n}+\frac{1}{2 h^{2}}\left(\psi_{-}-2 \psi_{n}+\psi_{+}\right)+\frac{1}{2}\left(\psi_{-}+\psi_{+}\right) \times\left[\frac{\alpha}{2}\left(\left|\psi_{-}\right|^{2}+\left|\psi_{+}\right|^{2}\right)+(1-\alpha)\left|\psi_{n}\right|^{2}\right]=0 .
$$

Notice that the integrable discretization of ref. [1] is obtained from this approach as the special case of $\alpha=0$. For $\alpha \neq 0$, this model can be regarded as a Salerno-type model [24], i.e., a homotopic continuation including the integrable limit and reducing to NLS equation in the continuum limit.

Expression (15) contains only the terms with even powers of $f_{-}$and $f_{n}$. However, it is possible to construct the terms of desired symmetry involving odd powers [8]. For example, for Kerr nonlinearity one can take

$$
G\left(f_{-}, f_{n}\right)=\frac{1}{4} f_{-} f_{n}\left(f_{-}^{2}+f_{n}^{2}\right),
$$

and obtain from Eqs. (10) and (12)

$$
-\omega f_{n}+\frac{1}{2 h^{2}}\left(f_{-}-2 f_{n}+f_{+}\right)+\frac{f_{n}^{3}}{4}+\frac{f_{n}}{4}\left(f_{-}^{2}+f_{-} f_{+}+f_{+}^{2}\right)=0,
$$


for which the following discretization can be obtained

$$
i \dot{\psi}_{n}+\frac{1}{2 h^{2}}\left(\psi_{-}-2 \psi_{n}+\psi_{+}\right)+\frac{1}{4}\left|\psi_{n}\right|^{2} \psi_{n}+\frac{1}{4}\left(\left|\psi_{-}\right|^{2}+\left|\psi_{-} \psi_{+}\right|+\left|\psi_{+}\right|^{2}\right) \psi_{n}=0
$$

The model of Eq. (23) has on-site cubic nonlinearity modified through inter-site coupling, which makes it qualitatively different from integrable system of [1]. Soliton solutions to this model can be constructed from the quartic Eq. (10) with $G$ given by Eq. (21). Note that DNLS equation with the quintic term possessing a structure similar to the last term of Eq. (23) has been considered in ref. [14] [see Eq. (74) of that paper].

\section{Momentum and Mass Conservation Laws}

We now try to connect the above presented construction to the relevant conservation laws of the resulting infinitedimensional dynamical system. More specifically, we examine the momentum conservation law that, as argued above, is intimately related to the translational invariance and the existence of mono-parametric stationary solutions. For the DNLS model of Eq. (3), we consider the momentum defined as

$$
P=i \sum_{n=-\infty}^{\infty}\left(\psi_{n} \psi_{+}^{\star}-\psi_{n}^{\star} \psi_{+}\right) \equiv i \sum_{n=-\infty}^{\infty} \psi_{n}\left(\psi_{+}-\psi_{-}\right)^{\star} .
$$

We now demand that the momentum be conserved, i.e., that

$$
\frac{\mathrm{d} P}{\mathrm{~d} t}=0
$$

Upon substitution of Eq. (24) and use of the equation of motion Eq. (3), $i \dot{\psi}_{n}+r_{n}=0$, for $\dot{\psi}_{n}, \dot{\psi}_{+}^{\star}$ and $\dot{\psi}_{-}^{\star}$, we obtain the following condition for conservation of the momentum

$$
\sum_{n=-\infty}^{\infty}\left[r_{n}^{\star}\left(\psi_{+}-\psi_{-}\right)+r_{n}\left(\psi_{+}^{\star}-\psi_{-}^{\star}\right)\right]=0 .
$$

A direct consequence of the above is that the regular DNLS equation will not succeed in leading to conservation of momentum, while it can easily be checked that the opposite is true for the model of Eq. (18) with $R$ given by Eq. (17).

The Eq. (18) with $R$ given by Eq. (17) conserves momentum Eq. (24) but it does not conserve the "standard" $\left(l^{2}\right)$ norm

$$
N=\sum_{n}\left|\psi_{n}\right|^{2}
$$

Instead Eq. (18) preserves the "mass" (norm) of the form:

$$
\tilde{N}=\sum_{n} \psi_{n}\left(\psi_{+}^{\star}+\psi_{-}^{\star}\right)
$$

which in the continuum limit retrieves the standard conservation law of the $L^{2}$ norm. Notice, however, that the discretization of Eq. (23) does conserve the standard norm.

For reasons of comparison that will become more transparent below, let us also introduce an additional discretization that does not belong to the family of Eq. (18) presented above:

$$
i \dot{\psi}_{n}+\frac{1}{2 h^{2}}\left(\psi_{-}-2 \psi_{n}+\psi_{+}\right)+\frac{1}{4}\left(\psi_{-}+\psi_{+}\right)\left|\psi_{n}\right|^{2}+\frac{1}{4}\left(\psi_{-}^{\star}+\psi_{+}^{\star}\right) \psi_{n}^{2}=0
$$

The particular feature of this dynamical system is that it conserves the $l^{2}$ norm and the momentum defined above. However, the standing wave ansatz of Eq. (4) reduces Eq. (29) to

$$
-\omega f_{n}+\frac{1}{2 h^{2}}\left(f_{-}-2 f_{n}+f_{+}\right)+\frac{1}{2}\left(f_{-}+f_{+}\right) f_{n}^{2}=0,
$$


which is particular case of Eq. (16) at $a_{2}=1 / 2, a_{1}=a_{3}=a_{4}=0$ and $\alpha=0$. Thus, solution of Eq. (30) can be found from the two-point difference equation

$$
u\left(f_{-}, f_{n}\right) \equiv \frac{1}{h^{2}}\left(f_{n}-f_{-}\right)^{2}-2 \omega f_{-} f_{n}+f_{-}^{2} f_{n}^{2}=0,
$$

which is a particular case of Eq. (10). As a result, the stationary states can be found by the solution of a reduced twopoint problem; namely, for any $f_{-}$(or $f_{n}$ ) in the range $\left[q_{m}, q_{s}\right]$, where $q_{m}=\sqrt{2 \omega}$ and $q_{s}=h^{-1} \sqrt{1-\left(1+\omega h^{2}\right)^{-2}}$, solving the binomial Eq. (31), one can find $f_{n}$ (or $f_{-}$), thus reconstructing the soliton solution for Eq. (29) in the form of Eq. (4). Quantities $q_{m}$ and $q_{s}$ are the amplitudes of solitons centered between two lattice sites and on a lattice site, respectively. Interestingly, $q_{m}$ is not a function of $h$. Even more interestingly perhaps, such stationary solutions of Eq. (29) are identical (see also below) to those of the integrable equation [Eq. (20) at $\alpha=0$ ] since the two models share the same reduced two-point problem [Eq. (31)].

\section{Comparison of soliton solutions for different discretizations}

We now compare some properties of the classical DNLS model of Eq. (19) (model I), the "secular" model of Eq. (29) conserving the classical norm (model II), and the Kerr-representative of the class of models developed herein, given by Eq. (20) (model III).

All three models share the same continuum limit, the integrable NLS equation with Kerr nonlinearity,

$$
i \psi_{t}+\frac{1}{2} \psi_{x x}+|\psi|^{2} \psi=0
$$

and thus, in the regime of weak discreteness (small lattice spacing $h$ ), their soliton solutions of the form of Eq. (4) can be expressed approximately as

$$
\psi_{n}(t)=\frac{q}{\cosh \left[q h\left(n-x_{0}\right)\right]} \exp [-i \omega t]
$$

where $q$ and $\omega=q^{2} / 2$ are the soliton amplitude and frequency, respectively.

The approximate solution of Eq. (33) contains the free parameter $x_{0}$ defining the soliton position. However, as indicated above, in contrast to the NLS equation of Eq. (32), where $x_{0}$ can be chosen arbitrarily due to translational invariance, the DNLS models usually have stationary soliton solutions only for a discrete set of values of $x_{0}$ (e.g., on-site and inter-site, as mentioned above). This is true, for example, for the classical DNLS of model I and for the Salerno model [24], among others. The models II and III, by construction, are among the members of a wider class of DNLS equations proposed in this paper, where stationary soliton solutions exist for any $x_{0}$, or, in other words, they can be placed anywhere with respect to the lattice; otherwise put, the static Peierls-Nabarro potential is absent for stationary solutions of these models.

An explicit formula, as is well-known [16], does not exist for the solutions of model I. Such solutions can be obtained numerically with the desired degree of accuracy, for two particular cases of $x_{0}=0$ and $x_{0}=1 / 2$ (due to the integer shift-invariance of the lattice, we now restrict ourselves to $0 \leq x_{0} \leq 1$ ). To obtain the soliton of frequency $\omega$ centered on a lattice site, i.e., the one with $x_{0}=0$, we set $f_{0}=\sqrt{2 \omega}$ which is the soliton amplitude estimated from Eq. (33). Having $f_{0}$ and the symmetry property $f_{n}=f_{-n}$ for $n>0$, we find successively $f_{1}$, then $f_{2}$ and so on from the equation $-\omega f_{n}+\left(2 h^{2}\right)^{-1}\left(f_{-}-2 f_{n}+f_{+}\right)+f_{n}^{3}=0$, which is obtained by substituting Eq. (4) into Eq. (19). Since $f_{0}$ is not an exact value of the soliton amplitude, the boundary conditions $f_{-\infty}=f_{\infty}=0$ will not be satisfied. We find numerically a correction to $f_{0}$ to satisfy the boundary conditions thus completing the construction of the soliton solution. For $x_{0}$ different from 0 or $1 / 2$ it is impossible to satisfy both boundary conditions simultaneously. The soliton with $x_{0}=1 / 2$ can be constructed similarly using the symmetry property $f_{-1}=f_{0}$ and $f_{n}=f_{-n-1}$ for $n>0$. Estimation of the soliton amplitude from Eq. (33) in this case is $f_{0}=\sqrt{2 \omega} / \cosh [(h / 2) \sqrt{2 \omega}]$. Alternatively, one can straightforwardly use fixed point algorithms to obtain such solutions as is summarized in ref. [16].

For the models II and III, the exact solutions of the form of Eq. (4) can be found using the method developed in Section 4. More specifically, however, for model II, as is expected from the discussion above and the coincidence of the reduced two-point problem with that of the integrable discrete model, an exact stationary solution can be obtained 
explicitly in the form

$$
\psi_{n}(t)=\frac{1}{h} \frac{\sinh \mu}{\cosh \left[\mu\left(n-x_{0}\right)\right]} \exp ^{i \omega t}
$$

where $x_{0}$ is the parameter defining the soliton position and it can obtain any value from $[0,1)$. The soliton frequency $\omega=h^{-2}(1-\cosh \mu)$ and amplitude $q=h^{-2} \sinh ^{2} \mu$ are expressed in terms of the free parameter $\mu>0$. The surprising feature of this secular discretization is that, despite the absence of an explicit momentum conservation law, the stationary solutions appear to enjoy an effective invariance with respect to their center location.

Model III has the solutions of the form of Eq. (4) with $f_{n}$ derivable from the two-point problem

$$
\frac{1}{h^{2}}\left(f_{-}-f_{n}\right)^{2}-2 \omega f_{-} f_{n}+\frac{\alpha}{2}\left(f_{-}^{4}+f_{n}^{4}\right)+(1-\alpha) f_{-}^{2} f_{n}^{2}=0 .
$$

Eq. (35) is a particular case of Eq. (10) with $G$ given by Eq. (15) at $a_{k}=0$ for all $k$ except for $a_{1}=1 / 2$. The soliton can be constructed by setting an arbitrary value for $f_{-}$(or $f_{n}$ ) in the range $\left[q_{m}, q_{s}\right]$ and finding $f_{n}$ (or $f_{-}$) from the quartic Eq. (35). Quantities $q_{m}$ and $q_{s}$ are the amplitudes of solitons centered between two lattice sites and on a lattice site, respectively. We have $q_{m}=\sqrt{2 \omega}$, which does not depend on $h$ and $\alpha$. For $f_{-}>q_{s}$ Eq. (35) does not have real solutions, i.e., $q_{s}$ corresponds to the magnitude of $f_{-}$for which the two distinct real roots of Eq. (35) in $f_{n}$ merge into a multiple root. The arbitrariness in the choice of initial value of $f_{-}$(or $\left.f_{n}\right)$ implies the absence of the Peierls-Nabarro potential and the possibility to place the soliton anywhere with respect to the lattice.

The dynamical evolution of $\left|\psi_{n}\right|^{2}$ for exact solitary wave solutions (constructed numerically as described above) in the classical DNLS of model I is shown in Fig. 1 where we compare the solitons centered at (a) $x_{0}=0$ and (b) $x_{0}=1 / 2$. The results are obtained by numerical integration of Eq. (19) for lattice spacing $h=0.4$ and soliton frequency $\omega=1$. The stationary solution in Fig. 1a is stable while the inter-site centered one in Fig. 1b is unstable; due to the presence of perturbations in the form of round-off errors, it spontaneously starts to alternate between the two nearest inter-site configurations passing through the stable on-site configuration. The pulse of Fig. 1a does not radiate while that of Fig. $1 \mathrm{~b}$ does.

Similar computations have been carried out in models II and III, and are reported in Fig. 2, also using $h=0.4$ and $\omega=1$. The solitary waves were found to be stable for any $x_{0}$ both in model II and model III for negative and positive $\alpha$ in a vicinity of $\alpha=0$. For example, in Fig. 2 we show the results for (a) model II and (b) model III with $\alpha=0.2$ and initial profiles placed non-symmetrically with respect to the lattice. In both cases the solitons do not radiate and they do not move to a higher-symmetry position.
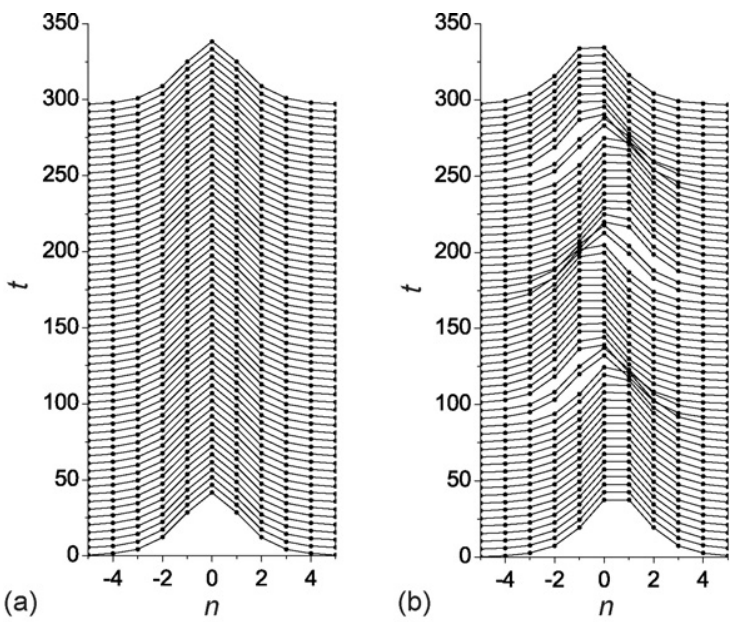

Fig. 1. The dynamical evolution of $\left|\psi_{n}\right|^{2}$ for exact solitary wave solutions in the classical DNLS model I centered at (a) $x_{0}=0$ and (b) $x_{0}=1 / 2$. Soliton in (a) is stable while in (b) it is unstable and, due to the presence of perturbations in the form of round-off errors, it spontaneously starts to alternate between two nearest inter-site configurations passing through the stable on-site configuration. Results for lattice spacing $h=0.4$ and soliton frequency $\omega=1$. 

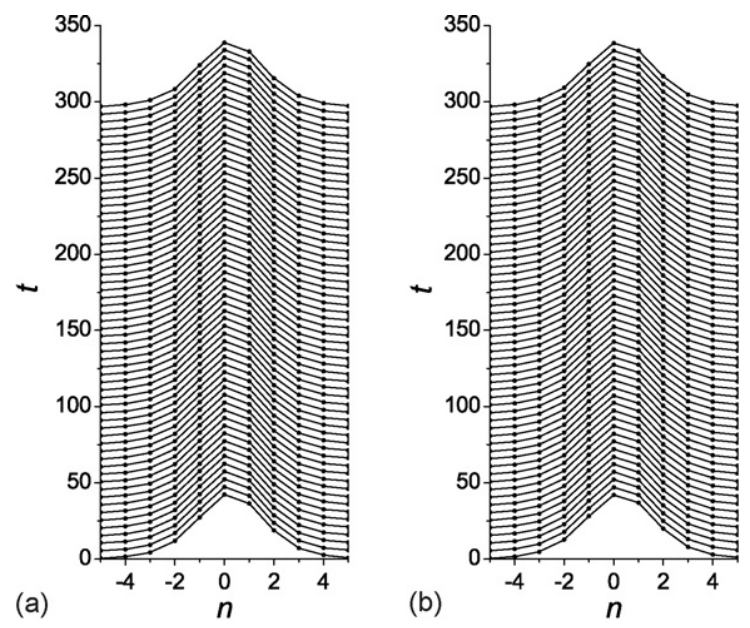

Fig. 2. The dynamical evolution of $\left|\psi_{n}\right|^{2}$ for exact solitary wave solutions placed non-symmetrically with respect to the lattice for (a) model II and (b) model III with $\alpha=0.2$. In both cases the solitons do not radiate and they do not move to a higher-symmetry position since the Peierls-Nabarro potential is absent. Results for lattice spacing $h=0.4$ and soliton frequency $\omega=1$.

\section{Conclusions}

We have described a general and systematic method of constructing spatial discretizations of NLS-type models, whose stationary soliton solutions can be obtained from a two-point difference problem. In this setting, finding stationary solutions becomes tantamount to solving simple nonlinear algebraic equations. We have also illustrated the connections of the resulting models with the integrable discretization of the NLS equation, of which they are a natural generalization for cubic nonlinearities (our construction was given for arbitrary polynomial nonlinearities of a particular parity); furthermore, the differences of such models from the standard discretization of the NLS equation often encountered in physical applications have been highlighted, both in terms of the relevant dynamical (solitonic) behavior as well as in terms of the underlying conservation laws present in the various models.

It would be particularly interesting to further examine such discretizations and their features, such as the stability of their solutions [16], and their travelling wave properties [4,11,21,7] and potential integrability of special members within these families. Such studies are currently in progress and will be reported in future publications.

\section{Note added in proof}

While this paper was under review, another work which establishes the momentum-conserving discretizations of the cubic discrete nonlinear Schrödinger equation appeared electronically in ref. [20].

\section{Acknowledgements}

We would like to acknowledge a number of useful discussions with Yu. S. Kivshar and also useful comments from D.E. Pelinovsky. SVD wishes to thank the warm hospitality of the Nonlinear Physics Centre at the Australian National University. PGK gratefully acknowledges the support of NSF-DMS-0204585, NSF-DMS-0505663 and NSF-CAREER.

\section{References}

[1] M.J. Ablowitz, J.F. Ladik, J. Math. Phys. 16 (1975) 598;

M.J. Ablowitz, J.F. Ladik, J. Math. Phys. 17 (1976) 1011.

[2] M.J. Ablowitz, B.M. Herbst, C. Schober, J. Comp. Phys. 126 (1993) 299.

[3] S. Aubry, Physica D 103 (1997) 201;

S. Flach, C.R. Willis, Phys. Rep. 295 (1998) 181;

D. Hennig, G. Tsironis, Phys. Rep. 307 (1999) 333. 
[4] I.V. Barashenkov, O.F. Oxtoby, D.E. Pelinovsky, Phys. Rev. E 72 (2005) 035602(R);

O.F. Oxtoby, D.E. Pelinovsky, I.V. Barashenkov, Nonlinearity 19 (2006) 217.

[5] C.M. Bender, A. Tovbis, J. Math. Phys. 38 (1997) 3700.

[6] D.N. Christodoulides, F. Lederer, Y. Silberberg, Nature 424 (2003) 817;

Yu.S. Kivshar, G.P. Agrawal, Optical Solitons: From Fibers to Photonic Crystals, Academic Press, San Diego, 2003.

[7] S.V. Dmitriev, P.G. Kevrekidis, A.A. Sukhorukov, N. Yoshikawa, S. Takeno, Phys. Lett. A 356 (2006) 324.

[8] S.V. Dmitriev, P.G. Kevrekidis, N. Yoshikawa, J. Phys. A 38 (2005) 7617;

S.V. Dmitriev, P.G. Kevrekidis, N. Yoshikawa, J. Phys. A 39 (2006) 7217.

[9] S.V. Dmitriev, P.G. Kevrekidis, N. Yoshikawa, D.J. Frantzeskakis, Phys. Rev. E 74 (2006) 046609.

[10] N.K. Efremidis, D.N. Christodoulides, Phys. Rev. E 65 (2002) 056607;

P.G. Kevrekidis et al., Physica D 183 (2003) 87.

[11] J. Gómez-Gardenes, F. Falo, L.M. Floría, Phys. Lett. A 332 (2004) 213;

J. Gómez-Gardenes, L.M. Floría, M. Peyrard, A.R. Bishop, Chaos 14 (2004) 1130.

[12] L. Hadziewski et al., Phys. Rev. Lett. 93 (2004) 033901.

[13] T. Kapitula, P.G. Kevrekidis, Nonlinearity 14 (2001) 533.

[14] P.G. Kevrekidis, Physica D 183 (2003) 68.

[15] P.G. Kevrekidis, D.J. Frantzeskakis, Mod. Phys. Lett. B 18 (2004) 173;

P.G. Kevrekidis, et al., Mod. Phys. Lett. B 18 (2004) 1481;

V.V. Konotop, V.A. Brazhnyi, Mod. Phys. Lett. B 18 (2004) 627.

[16] P.G. Kevrekidis, K.O. Rasmussen, A.R. Bishop, Int. J. Mod. Phys. B 15 (2001) 2833.

[17] S.J. Orfanidis, Phys. Rev. D 18 (1978) 3828.

[18] M. Öster, M. Johansson, A. Eriksson, Phys. Rev. E 67 (2003) 056606.

[19] L. Pilloni, D. Levi, Phys. Lett. A 92 (1982) 5.

[20] D.E. Pelinovsky, Nonlinearity 19 (2006) 2695.

[21] D.E. Pelinovsky, V.M. Rothos, Physica D 202 (2005) 16.

[22] M. Peyrard, Nonlinearity 17 (2004) R1.

[23] M. Peyrard, M. Kruskal, Physica D 14 (1984) 88.

[24] M. Salerno, Phys. Rev. A 46 (1992) 6856.

[25] J.M. Speight, Nonlinearity 12 (1999) 1373;

J.M. Speight, R.S. Ward, Nonlinearity 7 (1994) 475.

[26] J.M. Speight, Y. Zolotaryuk, Nonlinearity 19 (2006) 1365. 